BMJ Open Sport \& Exercise Medicine

\title{
The addition of a tension night splint to a structured home rehabilitation programme in patients with chronic plantar fasciitis does not lead to significant additional benefits in either pain, function or flexibility: a single- blinded randomised controlled trial
}

Patrick C Wheeler ${ }^{1,2,3}$

To cite: Wheeler PC. The addition of a tension night splint to a structured home rehabilitation programme in patients with chronic plantar fasciitis does not lead to significant additional benefits in either pain, function or flexibility: a single-blinded randomised controlled trial. BMJ Open Sport Exerc Med 2017;3:e000234.

doi:10.1136/bmjsem-2017000234

Accepted 3 May 2017

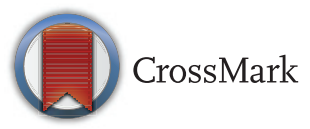

${ }^{1}$ Department of Sport \& Exercise Medicine, University Hospitals of Leicester NHS Trust, Leicester, UK ${ }^{2}$ School of Sport, Exercise and Health Sciences, Loughborough University, Loughborough, UK ${ }^{3}$ National Centre of Sport and Exercise Medicine - East Midlands (NCSEM), Loughborough, UK

Correspondence to Dr Patrick C Wheeler; patrick. wheeler@uhl-tr.nhs.uk

\section{ABSTRACT}

Objective To identify any improvements in pain or function in patients with chronic plantar fasciitis following the use of a tension night splint (TNS).

Methods Single-blinded randomised controlled trial, with participants split evenly between intervention group (TNS + home exercise programme/HEP) and control group (HEP only). Follow-up at 3 months, with interim data at 6 weeks.

Results 40 patients recruited. Mean age 52.1 years $33 \%$ male, mean body mass index $30.8 \mathrm{~kg} / \mathrm{m}^{2}$, mean duration of symptoms of 25 months. Improvement in self-reported 'average pain' in the intervention group from $6.8 / 10$ at baseline to $5.6 / 10$ at 6 weeks, and $5.3 / 10$ at 3 months (both clinically and statistically significant at both time points), compared with control group of $7.1 / 10$ at baseline to $6.2 / 10$ at 6 weeks and $5.6 / 10$ at 3 months (significant only at 3 months). Improvements in self-reported 'worst pain', 'pain walking' and 'pain first thing in the morning' in both groups at all time periods. Improvements were seen in revised Foot Function Index at all time points in both groups, but limited changes seen in flexibility and no significant changes in anxiety or depression Hospital Anxiety and Depression Scale domains or sleep quality in either group. However, no differences were seen between the outcomes seen in the two groups for the majority of the measures studied. Conclusions Improvements in pain and some functional measures seen in both groups, with few, if any, differences seen in outcomes between the intervention group compared with the control group. However, ongoing pain symptoms were reported in both groups, suggesting that 'help' rather than 'cure' was obtained for the majority. There is a possibility of earlier benefit seen in the intervention group compared with the control group, but data are unclear and further work may be needed.
Trial registration number ClinicalTrials.gov: NCT02546115; results.

\section{BACKGROUND}

Plantar fasciitis is a common condition causing under-surface heel pain. This most typically affects people age $40-60$, is more common in women than men and can affect both sedentary and active populations. ${ }^{1-3}$ Histological findings are typically that of myxoid degeneration, rather than inflammatory processes, and plantar fasciitis is

\section{What are the new finding?}

There is limited and inconsistent evidence for the use of tension night splints previously published, particularly in a population with chronic plantar fasciitis. This structured single-blinded study shows some benefits in pain and local function, albeit not in flexibility, in both of the groups studied, with a possibility of earlier benefit seen in the intervention group. The very limited differences in outcome seen between the groups questions the clinical effectiveness in outcomes in this population of the use of a tension night splint.

- Patients with even very chronic symptoms of plantar fasciitis have a significant improvement in various pain markers with a structured home exercise programme; however, it is not clear if the addition of a tension night splint device has much, if any, additional benefit beyond this.

- Assessment of flexibility seems to improve less than the reduction in pain suggests, indicating that benefits are seen directly, rather than just by improvements in soft-tissue flexibility. 
How might it impact on clinical practice in the near future?

This raises questions of the effectiveness of off-the-shelf tension night splint devices in patients with chronic plantar fascia pain. Patient benefit was seen in both groups, and limited, if any differences seen between groups. However, these data do strengthen the evidence for discussion with patients about expected benefits from either/both treatments in those with very chronic symptoms and suggest that some benefits in pain are possible with relatively simple interventions.

akin to an 'over-use' or 'under-recovery' type of condition, rather than a single acute injury. ${ }^{3-5}$ Due to the lack of an inflammatory-driven process, this condition is sometimes referred to as 'plantar fasciosis' or 'plantar fasciopathy' in some published works, but for the purposes of this study the name 'plantar fasciitis' will continue to be used as this remains its the most common form; however, the limitations of this term are recognised.

The plantar fascia itself is a tough band of connective tissue in the sole of the foot. It originates at the medial process of the tuberosity of the calcaneus and inserts in slips to the proximal phalanxes. It has a role in supporting the longitudinal arch of the foot, but also has roles in proprioception and peripheral motor coordination and contains Pacini and Ruffini corpuscles and nerve endings. ${ }^{6}$ Originating as it does from the under-surface of the calcaneus, the plantar fascia is sometimes viewed as the functional end point of the Achilles tendon, which is attached to the posterior margin of the calcaneus, and there are superficial fibres directly connecting the two structures which persist into adult life. ${ }^{7}$ Increasing tension in the Achilles tendon increases strain within the plantar fascia which may be a contributing factor to the development of plantar fasciitis although the evidence remains unclear. ${ }^{8} 9$ Tight posterior chain muscles (hamstrings, gastrocnemius, soleus) are often found in patients with plantar fasciitis, ${ }^{10}{ }^{11}$ with a reduction in ankle dorsiflexion being one of the most significant risk factors for the development of plantar fasciitis. ${ }^{12}$ Addressing any particular tightness in the calf muscle is therefore a key consideration in the management of patients with plantar fasciitis, with improvements in tightness correlating well to improvements in pain in these patients. ${ }^{13}$

Most patients with plantar fasciitis will improve within 12 months, but at least $10 \%$ may go on to develop persisting symptoms. ${ }^{2} 1415$ There is evidence of benefit from treatment with various low-risk conservative therapies including rest/activity modification, stretches and/or taping, ${ }^{13}{ }^{15-17}$ insoles/orthotics ${ }^{18}$ and possibly some evidence for the use of tension night splints (TNS) although this remains uncertain. ${ }^{19-26}$

Corticosteroid and local anaesthetic injections are often used in patients with plantar fasciitis, and a variety of differing techniques and broad outcome figures for success rates are reported in the literature; however, some papers report a plantar fascia rupture rate of up to $10 \%$ indicating that some caution may be needed. ${ }^{27-30}$ If these measures prove unsuccessful, then a range of more invasive options have been suggested including extracorporeal shockwave therapy, ${ }^{31}{ }^{32}$ autologous blood injections ${ }^{33-35}$ or surgery. ${ }^{36-38}$ However, comparing outcomes from these different options is often difficult due to the heterogeneity of patient populations, treatment interventions and follow-up, and many of these have not been subject to rigorously designed investigation.

Many different validated measures exist both for specific foot pain and wider function. Specific foot measures include forms of the Foot Function Index/ Revised Foot Function Index (FFI/FFI-R), which is a self-rating questionnaire with good test-retest reliability, which has moderate-to-high levels of correlation with the 36-Item Short Form Health Survey (SF-36). ${ }^{39-41}$ The 16-item Manchester-Oxford Foot Questionnaire (MOXFQ) has been validated against both the SF-36 and the American Orthopaedic Foot and Ankle Score (AOFAS) questions for a wide range of foot and ankle problems, ${ }^{42-44}$ with further work showing validity from a combined, single score rather than the original subscales. ${ }^{45}$ The Foot and Ankle Ability Measure (FAAM) questionnaire has also been found to be valid, with high correlation with the SF-36 physical function subscale. ${ }^{46}$ Wider aspects of function which are important to consider include global health function which can be assessed by the EuroQol five dimensions questionnaire (EQ-5D) which has good validity across a number of populations and the ' $5 \mathrm{~L}$ ' version reduces the ceiling effect seen in previous versions of the questionnaire. ${ }^{47}$ For simplicity, the global self-reported percentage health score is included in this study to give an overall indication of health, with a higher score indicating better self-rated overall health. Other considerations include the possibility of the presence of neuropathic pain, sleep disturbance and mental health functioning, which may all influence and be influenced by overall pain levels. The Hospital Anxiety Depression Scale (HADS), which has been validated across a range of populations, gives 0-21 subscales for anxiety and depression symptoms, with a higher score indicating more symptoms. ${ }^{48}{ }^{49}$ Sleep disturbance is another issue which is common in patients with pain from a range of sources, and anecdotally patients often report that the TNS device can adversely affect sleep quality. To measure sleep quality, the Pittsburgh Sleep Quality Index (PSQI) was used in this study. This gives a measure of sleep quality across a range of subscales, and for simplicity the total score 
is included here across a 0-21 scale, with higher value indicating poorer sleep quality. ${ }^{50} 51$

The painDETECT questionnaire was originally developed for the assessment of patients with chronic low back pain and has high sensitivity, specificity and positive predictive value for the presence of neuropathic pain in a prospective multicentre trial. ${ }^{52}$ Following this the questionnaire has been used in a wide range of settings with differing clinical problems, ${ }^{53} 54$ and there are thoughts that some patients with chronic tendinopathy may also score positively for neuropathic pain using painDETECT, the significance of which remains unclear currently. ${ }^{55}$ Scoring 19-38 suggests a $90 \%$ likelihood of a neuropathic pain component to a patient's symptoms, with $13-18 / 38$ being an ambiguous result and $0-12 / 38$ suggests that neuropathic pain component is unlikely $(<15 \%)$. Identification of those patients with a previously unknown neuropathic pain component may allow alternative treatments, or treatments alongside that for the nociceptive component of pain.

The use of a TNS device to assist in the stretching of the calf muscle complex has a plausible rationale for use and benefit in the treatment of patients with chronic plantar fasciitis. However, there is limited published evidence of benefit to date of this intervention, despite it being in routine clinical use. A small case series, published in 1991, suggested benefit from the use of night splints in patients with recalcitrant plantar fasciitis. ${ }^{19}$ Following this, a small prospective randomised control trial (RCT published in 1996 showed improved outcomes in a group treated with night splint alongside other treatments which included stretching, Non-Steroidal Anti-Inflammatory Drugs (NSAIDs) and a heel cup, compared with a group without a splint, using clinical outcome measures. ${ }^{20}$ However, a larger prospective RCT published in 1999 showed no difference in outcomes of treatment with the addition of a TNS with clinical outcome and SF-36 used as outcome measures. ${ }^{21}$ A different crossover study published in 1998 and which used the AOFAS ankle-hindfoot rating and the Mayo Clinical Scoring System demonstrated improvements with the addition of the splint devices. ${ }^{22}$ One large study in 2001 compared two different orthotic devices and a TNS device, and found overall no differences between the groups in terms of outcome, but a higher discontinuation rate in the TNS group. ${ }^{23}$ A retrospective study sought to compare the outcomes from simple stretchbased programme and those from the use of a TNS, and this found quicker recovery time and fewer further treatments were required in the TNS group, although the retrospective nature of this study and the lack of randomisation means that these results are subject to greater bias than other studies. ${ }^{24} \mathrm{~A}$ small case series of 12 patients using a specific TNS device was published in 2002 suggesting good results with both pain and a functional scale and high levels of tolerability form this device, but the small study size and lack of control or comparison group limits the impact of this research. ${ }^{25}$ Lastly, a retrospective study based in the same department as this study found that patients reported good improvements in pain following the use of a TNS, but that $30 \%$ has ceased wearing the splint within 1 month from a variety of factors. However, the retrospective nature of this study and the lack of a control group again makes reliable assessment from this study difficult. ${ }^{26}$ A systematic review published in 2015, but using a search from November 2013, demonstrated that overall there was uncertain evidence for the use of TNS devices to treat patients with plantar fasciitis. ${ }^{56}$

Given this uncertainty, this study seeks to investigate the outcomes of a modern commercially available night splint in patients with chronic plantar fasciitis in a rigorous manner, using broad and reliable outcome measures of pain and function, to better determine its use in this condition in routine use.

\section{METHODS}

Patients who were being treated by the author in their clinic for symptoms of plantar fasciitis, and who had not benefited from a home exercise programme (HEP) already, were assessed for suitability to enter the study and if the inclusion/exclusion criteria were met. Baseline data were recorded by the author including baseline patient-rated outcome measures (PROMs) and measures of baseline flexibility, both of which are detailed below. Participants were then reviewed by the study nurse practitioner who advised them about their group allocation, and taught the participants in the intervention group how to apply the TNS device. Participants were then followed up at 6 weeks and 3 months by the study author, and repeat measurements were taken at each appointment. The author remained blind to treatment allocation until after the participants completed the 3-month follow-up assessment, and no accidental unblinding occurred during the study.

\section{Inclusion/exclusion criteria}

Patients were required be over the age of 18 , to have had unilateral plantar fasciitis symptoms only for at least 4 months, with pain reproduced on palpation on the medial calcaneal tubercle at the plantar fascia attachment, and with pain on loading, and have the diagnosis confirmed on either ultrasound (plantar fascia thickening of $>4 \mathrm{~mm}$, without full/partial thickness tear) or MRI. ${ }^{57}$ Exclusion criteria included previous or current partial or full thickness tears of the Achilles or plantar fascia (to avoid risk of further injury), lower limb sensorimotor or vascular problems (as these could be adversely affected by the use of the splint), those with other causes for their pain (such as referred pain, or inflammatory/connective tissue disorders) or those that could not adequately apply the splint device. Suitable patients who met the criteria had the study discussed with them, were given written 
information about the study and if they wished to participate were booked in to commence formal enrolment.

\section{Consent and randomisation}

Participants were consented to enter the study and randomised by a study nurse practitioner who remained independent from the rest of the study. Participants were allocated equally to either group A (the 'intervention group', who received a HEP and TNS) or group B (the 'control group', who received a HEP only) using the 'Sealed Envelope' software (www.sealedenvelope.com, Sealed Envelope)

\section{Intervention}

Both groups were given a structured HEP which included static stretches of the plantar fascia, the calf (selectively involving both gastrocnemius and soleus for different stretches), plus Flexor Hallucis Longus (FHL) and hamstrings, as well as calf and intrinsic foot muscle strengthening and balance training exercises. All participants were taught how to perform and progress their HEP themselves based on their progress at baseline. Their technique was checked at the interim (6 week) appointment and any necessary corrections made. All patients were also given written material to support the use of this HEP.

In addition to the HEP, participants who were randomised to group A (intervention group) were also given a commercially available TNS device (LA Brace Plantar Fasciitis Night Splint) which was bought from a medical supply company before the study commenced. Patients were taught how to apply the device by the study nurse practitioner, and had her contact details should any further assistance about the device be required. They were instructed how to alter the tension within the splint device over time both for comfort and to maintain a dorsiflexion force during the intervention period. Figure 1 is a picture of the TNS device used in this study.

\section{Data collection}

Patients completed a structured questionnaire about their symptoms before treatment commenced and at each the 6 week and 3-month follow-up visits. These outcome measures include questions about pain, as well as a range of validated PROMs which include questionnaires about local function (FFI-r and MOXFQ, in addition to measures of global function (EQ-5D-5L). Questionnaires are also used to examine the impact of symptoms of other areas of functioning including measures of anxiety and depression symptoms (HADS) and sleep quality (PSQI). In addition, questionnaires are used to quantify levels of physical activity. These include the short-form (7-day recall) version of the International

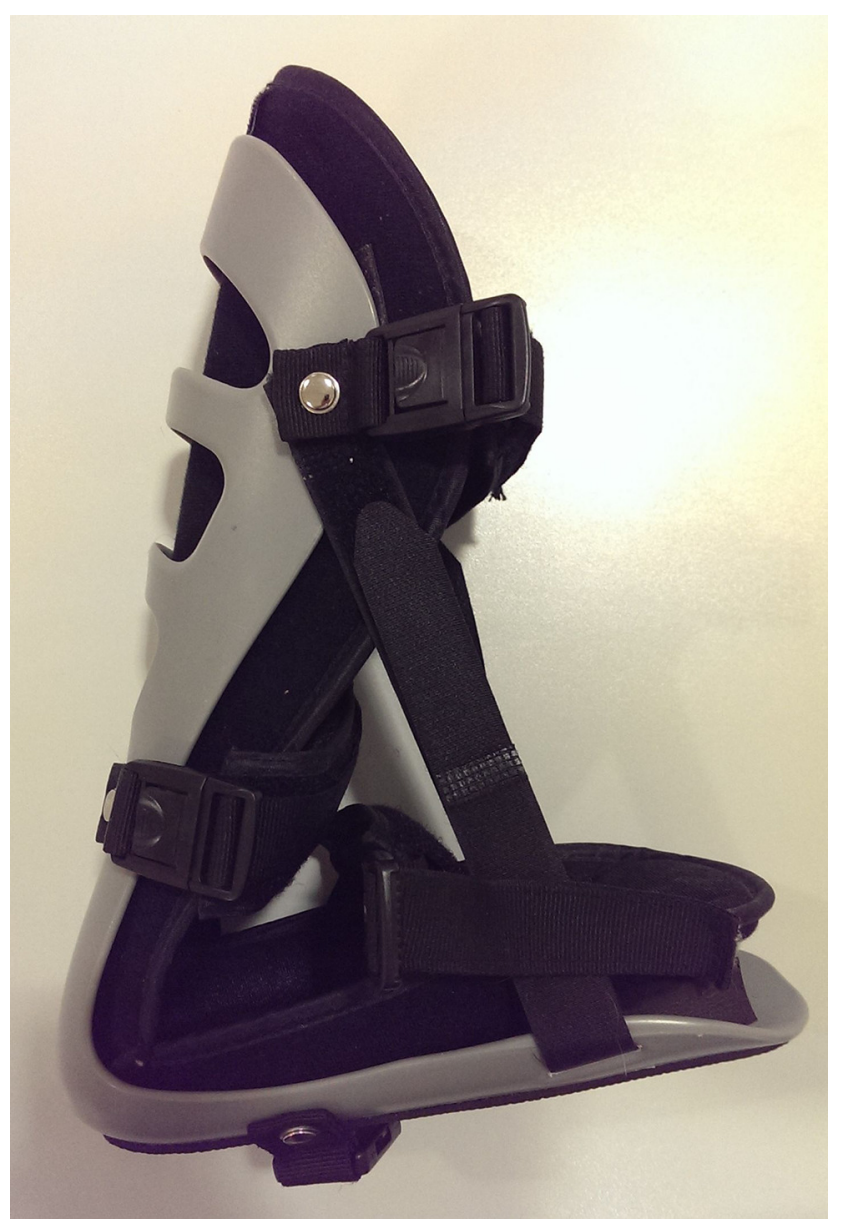

Figure 1 Tension night splint device.

Physical Activity Questionnaire, and two 'vital signs' physical activity questions.

In addition, physical assessments of flexibility were taken by the lead investigator using a hand-held goniometer (the range of passive ankle dorsiflexion with the subject in a prone position with the knee both extended and flexed to $90^{\circ}$, and an assessment of popliteal angle with the subject lying supine, with the hip flexed to $90^{\circ}$, and an assessment of the range of knee extension with $180^{\circ}$ being fully extended). The 'knee-to-wall' distance was recorded as a measure of flexibility in a more functional position, measuring the distance in millimetres a subject could move their foot back from the wall and still just touch the knee to the wall without the heel lifting. In addition, an assessment of plantar fascia thickness was recorded using a musculoskeletal ultrasound (GE LogiqE) with an average of three recordings made to reduce intraobserver error between measures, with previous work showing a reduction in plantar fascia thickness correlated to improvement in symptoms. ${ }^{58}$

The primary outcome measure studied was a change in average pain (as recorded on a $0-10$ scale) between baseline and at 3 months, with the remainder of the outcome measures studied being secondary measures to this. Those participants who were in the control group and 


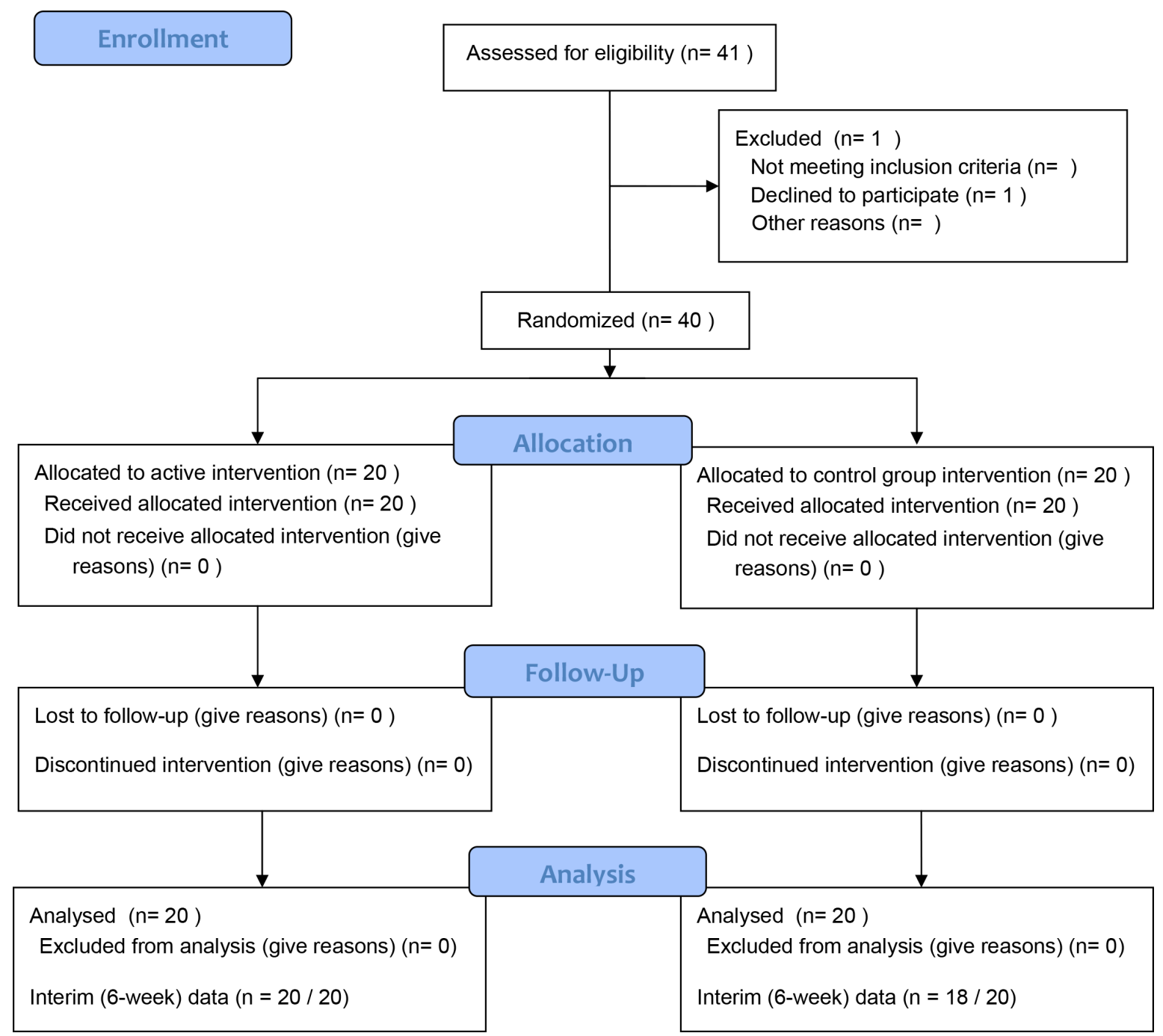

Figure 2 CONSORT flowchart.

who had not improved significantly at 3 months were offered a TNS after the final study follow-up.

\section{Ethical permissions}

This study has all necessary permissions and is registered on a publicly accessible database (ClinicalTrials.Gov identifier NCT02546115). The study was given a favourable opinion from an NHS Research Ethics Committee (REC ref 14/WS/1069) and has local site approvals.

\section{Statistical analysis}

An a priori power calculation was performed using available data for improvements in self-reported pain. This indicated that a range of between 15 and 25 participants in total were needed based on previously published pre/post differences. Allowing for possible drop-outs permissions were granted to recruit a total of 40 participants, split evenly between the intervention and control groups.

Data were recorded prospectively at baseline, and on an ongoing basis at clinic follow-up. This was collated into an Excel spreadsheet (MS Excel from MS Office
2011, V. 14.5.7) and analysed in SPSS (IBM SPSS Statistics, V. 23).

Comparisons were made between the baseline data and data from the 6 week, and the 3-month follow-up appointments, with most of the outcome measures in this dataset being scale data. The Shapiro-Wilk test was used to assess normality as the sample sizes were small. As most the data were found to be normally distributed, the analysis was predominantly performed with parametric testing, typically paired-samples t-test for comparison within groups, and independent-samples ttest for comparison between groups. Statistical significance was set at $\mathrm{p}<0.05$. All data were analysed on an intention-to-treat basis, deliberating not accounting for the amount of time patents wore the device, mimicking the effect in the real world.

\section{RESULTS}

A total of 41 participants with chronic plantar fasciitis treated by the author in their hospital clinic were initially identified as being potentially suitable for inclusion. One patient was initially put forward to enter 
the study, but after baseline assessment chose not to continue the study, leaving a total of 40 patients recruited. All patients completed the study with final data at 3 months; however, two patients did not attend for the interim/6-week appointment giving 38/40 responses at this point (one was due to an administrative error, the other due to patient availability) and both of these participants were in group B. Information for this is detailed in the included CONSORT 2010 flow diagram (figure 2).

There was mean age of the participants of 52.1 years; $33 \%$ were male. At entry into the study, the participants had a mean duration of symptoms of 25.2 months, and had a mean body mass index of $30.8 \mathrm{~kg} /$ $\mathrm{m}^{2}$. There were no statistically significant differences in any of the demographics recorded between participants in group A (intervention) and group B (control), and these figures are displayed in table 1, with figures displayed as mean (SD).

\section{Patient-rated outcome measures}

There were no differences in any of the baseline PROMs studied between the members of the two groups, except for the self-reported 'worst pain' which did differ significantly at baseline (group A/intervention $=8.0 / 10$, group $\mathrm{B} /$ control $=9.0 / 10, p=0.032)$, and it was not clear if this could have influenced subsequent analysis.

The baseline and follow-up variables for both group A and group B are displayed in table 2, figures shown are mean (SD), significance was calculated between group $\mathrm{A}$ and $\mathrm{B}$ at the same time point (column), and between the follow-up period and the baseline for both groups (row). (* indicates statistically significant change, but specific $\mathrm{p}$ values are not displayed to aid clarity). While improvements were seen in a number of the measures studied in members in both groups,

Table 1 Demographic information for subjects

\begin{tabular}{|c|c|c|c|}
\hline & $\begin{array}{l}\text { Group A } \\
\text { Intervention } \\
\text { group } \\
(n=20) \\
\text { HEP+TNS }\end{array}$ & $\begin{array}{l}\text { Group B } \\
\text { Control } \\
\text { group } \\
(n=20) \\
\text { HEP alone }\end{array}$ & p Value \\
\hline Age & $53.4(8.9)$ & 50.9 (11.7) & 0.444 \\
\hline $\begin{array}{l}\text { Gender (\% } \\
\text { male) }\end{array}$ & $25 \%$ & $35 \%$ & 0.503 \\
\hline BMI $\left(\mathrm{kg} / \mathrm{m}^{2}\right)$ & $32.6(5.0)$ & $29.0(4.9)$ & 0.071 \\
\hline $\begin{array}{l}\text { Symptom } \\
\text { duration } \\
\text { (months) }\end{array}$ & $30.3(33.2)$ & $20.1(9.2)$ & 0.204 \\
\hline
\end{tabular}

BMI, body mass index; HEP, home exercise programme;

TNS, tension night splint. including pain, and specific local foot function, there was no statistically significant differences seen between the two groups studied except for the sport subscore of the FAAM questionnaire favouring the control group, the significance of this was unclear.

\section{Physical parameters}

In group A (intervention group), there were statistically significant improvements in flexibility measured with the knee-to-wall test at 6 weeks only, but not at 3 months. In addition, significant changes were seen in the popliteal angle at 3 months, but not at 6 weeks. There were no significant changes in flexibility seen in group B at any of the time points studied. However, when comparing the groups at each time point, none of the physical parameters of flexibility measured reached a statistical significant difference between group A and group B at any of the time points studied.

There were no significant differences in plantar fascia thickness between members of group A and group $\mathrm{B}$ and baseline or either of the follow-up periods studied. In the members of group B only, the plantar fascia thickness decreased to a statistical significant amount between values recorded at baselines and those at both the 6 -week and 3-month time points; the clinical significance of this is uncertain.

The figures for the physical parameters measured are displayed in table 3 , figures displayed are mean (SD).

\section{DISCUSSION}

In keeping with previous published research, this study has shown improvements in several domains of pain and self-reported function in patients with the HEP both with and without the TNS. It is important to note that these benefits were seen in patients with very chronic symptoms, with an average of 2 years, which is far longer premorbid symptoms than many other previously published works. ${ }^{59}$ It is not clear if this duration of prior symptoms has had a direct effect on the outcomes seen, as most patients with plantar fasciitis symptoms will improve within 6 to 12 months, and there may be better results from earlier treatments, although this remains unclear. ${ }^{1}$

\section{Pain/PROMs results}

Members of both groups had statistically significant improvements in self-reported pain levels of pain of approximately 1.5 points on a $0-10$ pain scale at 3 months, which exceeded the minimally important differences of the equivalent of 0.9 points found in previous research. ${ }^{60}$ However, there appeared to be little, if any, significant difference between the two groups seen at any time point suggesting minimal additional benefit from the addition of a TNS device to a structure HEP. The data were all analysed on 
Table 2 Patient-rated outcome measures (PROMs) at baseline and follow-up

\begin{tabular}{|c|c|c|c|c|}
\hline PROMs/group & & Baseline & 6 weeks & 3 months \\
\hline \multirow{2}{*}{$\begin{array}{l}\text { 'Average pain' } \\
(0-10)\end{array}$} & $A$ & $6.8(2.2)$ & $5.6(2.8)^{\star}$ & $5.3(2.6)^{\star}$ \\
\hline & B & $7.1(1.7)$ & $6.2(2.6)$ & $5.6(2.9)^{\star}$ \\
\hline \multirow{2}{*}{$\begin{array}{l}\text { 'Pain at its worst' } \\
(0-10)\end{array}$} & A & $8.0(1.7)$ & $6.6(2.6)^{\star}$ & $5.9(2.9)^{\star}$ \\
\hline & $B$ & $9.0(1.0)^{\star}$ & $7.1(2.6)^{\star}$ & $6.7(3.2)^{\star}$ \\
\hline \multirow{2}{*}{$\begin{array}{l}\text { 'Pain at its best' } \\
(0-10)\end{array}$} & $A$ & $5.2(2.4)$ & $4.2(2.5)^{\star}$ & $3.7(2.2)^{\star}$ \\
\hline & B & $5.5(2.4)$ & $4.4(3.0)$ & $4.4(3.0)$ \\
\hline \multirow{2}{*}{$\begin{array}{l}\text { 'Pain in the morning' } \\
(0-10)\end{array}$} & $A$ & $7.3(2.2)$ & $5.8(2.6)^{\star}$ & $5.2(2.7)^{\star}$ \\
\hline & B & $8.3(1.3)$ & $6.8(2.9)^{\star}$ & $5.8(3.4)^{\star}$ \\
\hline \multirow{2}{*}{$\begin{array}{l}\text { 'Pain in the evening' } \\
(0-10)\end{array}$} & A & $6.9(2.1)$ & $6.1(2.9)$ & $5.5(2.8)^{\star}$ \\
\hline & B & $7.7(1.4)$ & $5.9(3.2)^{\star}$ & $6.0(3.1)^{\star}$ \\
\hline \multirow{2}{*}{$\begin{array}{l}\text { 'Pain at rest' } \\
(0-10)\end{array}$} & A & $5.4(2.7)$ & $4.3(2.9)^{\star}$ & $4.3(2.4)^{\star}$ \\
\hline & B & $6.0(2.2)$ & $5.2(2.7)$ & $4.9(3.0)$ \\
\hline \multirow{2}{*}{$\begin{array}{l}\text { 'Pain when walking' } \\
(0-10)\end{array}$} & A & $7.1(2.3)$ & $5.7(2.8)^{\star}$ & $5.5(2.8)^{\star}$ \\
\hline & B & $7.5(1.8)$ & $5.6(3.2)^{\star}$ & $5.5(3.1)^{\star}$ \\
\hline \multirow{2}{*}{$\begin{array}{l}\text { 'Average stiffness' } \\
(0-10)\end{array}$} & A & $6.0(3.2)$ & $4.9(2.9)$ & $4.3(2.9)^{\star}$ \\
\hline & B & $6.5(3.0)$ & $6.1(2.6)$ & $4.6(3.2)^{\star}$ \\
\hline \multirow{2}{*}{$\begin{array}{l}\text { 'Stiffness in the morning' } \\
(0-10)\end{array}$} & A & $6.7(3.0)$ & $5.3(2.9)$ & $4.9(3.0)$ \\
\hline & B & 5.7 (3.2) & 6.3 (3.2) & $4.6(3.6)$ \\
\hline \multirow{2}{*}{$\begin{array}{l}\text { painDETECT } \\
\text { (0-38, lower score indicating lower likelihood of neuropathic pain) }\end{array}$} & A & $13.4(8.6)$ & $11.4(7.3)$ & $11.9(8.0)$ \\
\hline & $\mathrm{B}$ & $15.4(7.3)$ & $13.6(7.5)$ & $11.9(5.2)^{\star}$ \\
\hline \multirow{2}{*}{$\begin{array}{l}\text { MOXFQ (total score) } \\
\text { (range } 16-48, \text { lower score=better function) }\end{array}$} & A & $56.2(14.5)$ & $50.3(16.0)^{\star}$ & $49.2(16.2)^{\star}$ \\
\hline & B & $64.5(12.0)$ & $58.4(16.7)$ & $51.8(17.2)^{\star}$ \\
\hline \multirow{2}{*}{$\begin{array}{l}\text { FFI-R (total) } \\
\text { (range 34-136, with lower score indicating better function) }\end{array}$} & A & $88.3(24.5)$ & $81.4(24.0)$ & $77.6(25.4)^{\star}$ \\
\hline & B & 95.5 (22.9) & $86.6(22.5)$ & $77.0(27.4)^{\star}$ \\
\hline \multirow{2}{*}{$\begin{array}{l}\text { FAAM-\%score } \\
\text { (higher score = better function) }\end{array}$} & A & $56 \%(23 \%)$ & $58 \%(23 \%)$ & $67 \%(22 \%)^{\star}$ \\
\hline & B & $55 \%(20 \%)$ & $61 \%(19 \%)^{*}$ & $66 \%(23 \%)^{*}$ \\
\hline \multirow{2}{*}{$\begin{array}{l}\text { FAAM-\% - function } \\
\text { (higher score indicating better function) }\end{array}$} & $A$ & $56 \%(28 \%)$ & $61 \%(25 \%)$ & $53 \%(27 \%)$ \\
\hline & $\mathrm{B}$ & $61 \%(13 \%)$ & $65 \%(26 \%)$ & $68 \%(22 \%)^{*}$ \\
\hline \multirow{2}{*}{$\begin{array}{l}\text { FAAM-\%sport score } \\
\text { (higher score indicating better function) }\end{array}$} & $A$ & $36 \%(27 \%)$ & $39 \%(29 \%)$ & $44 \%(31 \%)$ \\
\hline & B & $34 \%(22 \%)$ & $43 \%(20 \%)$ & $44 \%(31 \%)$ \\
\hline \multirow{2}{*}{$\begin{array}{l}\text { FAAM-\%sport function } \\
\text { (higher score indicating better function) }\end{array}$} & $A$ & $34 \%(32 \%)$ & $31 \%(25 \%)$ & $37 \%$ (27\%) \\
\hline & B & $51 \%(28 \%)$ & $53 \%(30 \%)^{\star}$ & $67 \%(28 \%)^{\star}$ \\
\hline \multirow{2}{*}{$\begin{array}{l}\text { HAD-anxiety subscale } \\
\text { (range 0-21, with lower score indicating fewer symptoms) }\end{array}$} & $A$ & $6.5(4.7)$ & $6.1(3.5)$ & $5.5(4.0)$ \\
\hline & $\mathrm{B}$ & $7.2(5.0)$ & $8.1(4.6)$ & $6.7(4.9)$ \\
\hline \multirow{2}{*}{$\begin{array}{l}\text { HAD-depression subscale } \\
\text { (range } 0-21 \text {, with lower score indicating fewer symptoms) }\end{array}$} & A & $5.7(4.6)$ & $6.6(5.1)$ & $5.6(4.6)$ \\
\hline & B & $6.1(4.7)$ & $5.8(4.2)$ & $5.1(4.7)$ \\
\hline \multirow{2}{*}{$\begin{array}{l}\text { PSQI (total score) } \\
\text { (range 0-21, with lower score indicating fewer symptoms) }\end{array}$} & A & $8.7(5.3)$ & $10.4(5.1)$ & $8.8(4.7)$ \\
\hline & $\mathrm{B}$ & $10.1(4.9)$ & $10.7(5.3)$ & $10.2(4.7)$ \\
\hline
\end{tabular}

Figures are mean(SD).

*Indicates statistically significant change from baseline for variable in row, or difference between group A and B for variable in column ( $p$ values not displayed for clarity).

FAAM, Foot and Ankle Ability Measure; FFI-R Revised Foot Function Index; HAD, Hospital Anxiety and Depression; MOXFQ, ManchesterOxford Foot Questionnaire; PSQI, Pittsburgh Sleep Quality Index. 
an intention-to-treat basis, which could have reduced any effects of the TNS, but this better represents the effects seen in the real world with some patients having limited compliance to the use of the device for a variety of factors including comfort. In addition, it is noted that the majority of participants were 'helped' rather than 'cured' within both groups, and realistic advice needs to be given to patients about expectations from treatment for those with chronic symptoms.

There was a statistically significant improvement in the intervention group of self-reported 'average pain' from baseline to 6 weeks of 1.2 points, and 1.5 points at 3 months, which is compared with the selfreported 'morning pain' improvements of 1.5 and 2.1 points, respectively, for self-reported pain first things in the morning. This indicates that pain is improved throughout the day with the HEP and TNS, although the lack of differences between groups suggests that the benefits seen may not be attributed to the TNS device.

The control group also achieved significant improvements in a range of pain measures including self-reported average pain, worst pain, pain in the morning, pain in the evening and pain when walking. This was despite them only having the same interventions within the study as they had before entry and the reasons for this remain unclear. It is possible that benefits were gained as patients had better compliance with the exercise programme within the study (the so-called Hawthorne effect'; however, this cannot be reliably determined from these data. Alternatively, it is possible that these benefits were a simply as a consequence of the natural resolution of the condition, but as the average duration of symptoms in the subjects in this group was in excess of 18 months, it is not clear whether the benefits seen over a further 3-month period could be solely attributable to this, and the mechanisms of benefits seen are unclear

Improvements in foot function were seen in both members of the intervention and control group, with improvements in the MOXFQ score reaching statistical significance in the intervention group at both 6 weeks and 3 months, but only at 3 months in the control group. This effect was reversed for the FAAM questionnaire score which showed statistical significant improvements in the intervention group at 3 months, but at the 6 -week and 3-month point for the control group. The FFI-R score changed to a statistically significant amount in both groups at the 3-month time point, although it did not differ significantly between the groups, and the magnitude of the improvements seen is greater than the seven points found to be the minimum clinically significant change in previous work. ${ }^{60}$ Overall, regarding specific foot function PROMs, while some benefits were seen, interpretation of any benefit being attributed to the TNS is unclear.

Although improvements in pain were seen in both groups, there was no significant improvement in mental health functioning for either anxiety or depressive symptoms as assessed by the HAD questionnaire. Somewhat reassuringly, although anecdotally patients may report impaired sleep quality from the use of the TNS devices, there was no statistically significant worsening of sleep quality as assessed by the PSQI, in members of the intervention group at either of the follow-up time periods studied.

\section{Physical parameters}

Despite the improvements in pain seen, less definite benefits were seen in either group across the domains of physical flexibility. Improvements were

Table 3 Physical measurements at baseline and at follow-up

\begin{tabular}{|c|c|c|c|c|}
\hline Physical parameters/group & & Baseline & 6 weeks & 3 months \\
\hline \multirow[t]{2}{*}{ Knee-to-wall distance $(\mathrm{mm})$} & A & $89.0(36.5)$ & $100.3(33.2)^{\star}$ & $93.4(37.0)$ \\
\hline & B & $86.8(41.5)$ & $89.4(35.9)$ & $97.3(39.1)$ \\
\hline \multirow[t]{2}{*}{ Ankle dorsiflexion angle-with knee straight } & A & $93.7^{\circ}\left(5.5^{\circ}\right)$ & $94.5\left(5.0^{\circ}\right)$ & $95.1\left(5.9^{\circ}\right)$ \\
\hline & $\mathrm{B}$ & $94.4^{\circ}\left(5.5^{\circ}\right)$ & $96.8\left(4.5^{\circ}\right)$ & $94.9\left(5.8^{\circ}\right)$ \\
\hline \multirow[t]{2}{*}{ Ankle dorsiflexion angle-with knee bent } & A & $99.2^{\circ}\left(7.0^{\circ}\right)$ & $101.2\left(6.4^{\circ}\right)$ & $99.5\left(5.3^{\circ}\right)$ \\
\hline & B & $98.6^{\circ}\left(5.1^{\circ}\right)$ & $100.7\left(5.7^{\circ}\right)$ & $99.8\left(5.5^{\circ}\right)$ \\
\hline \multirow[t]{2}{*}{ Popliteal angle } & A & $145.9^{\circ}\left(10.3^{\circ}\right)$ & $145.7\left(21.1^{\circ}\right)$ & $150.8\left(11.0^{\circ}\right)^{*}$ \\
\hline & B & $145.8^{\circ}\left(10.7^{\circ}\right)$ & $150.2\left(9.7^{\circ}\right)$ & $147.8\left(12.7^{\circ}\right)$ \\
\hline \multirow[t]{2}{*}{ Plantar fascia thickness (mm) } & A & $6.1(1.4)$ & $5.7(1.5)$ & $6.0(1.9)$ \\
\hline & $\mathrm{B}$ & $6.3(1.2)$ & $5.8(1.1)^{\star}$ & $5.7(1.2)^{\star}$ \\
\hline
\end{tabular}

*Indicates significant change from baseline for variable in row.

Figures shown are mean (SD). 
found in two measures in the intervention group, but no significant differences were seen between different groups, and neither group showing consistent improvements in flexibility. This differs from previous work showing a close correlation between the two variables. ${ }^{13}$

The plantar fascia thickness improved to a statistically significant extent in the control group, but not the intervention group. The reasons for this, and any clinical significance for the changes seen, remain unclear, with previous published works showing a correlation between reduction in plantar fascia thickening and improvements in pain which was not seen specifically in this study. ${ }^{58}$

\section{Comparisons}

The two groups were believed to be similar across a range of measurements at baseline, and while patients in the intervention group were slightly older, slightly more overweight and with a longer duration of symptoms, none of these variables reached statistical significance. Of the pain/function variables, the participants in the control group did have a statistically higher self-reported 'worst pain' at baseline, although the other variables were the same as the intervention group, and it is not clear if this could have had an influence on outcome results.

While improvements were seen in pain and local function in both groups at 3 months, the intervention group who received the HEP and the TNS device could possibly be considered to have improved quicker with statistically significant improvements in a range of outcome measures of pain at 6 weeks, whereas the control group, who received the HEP only, tended to require the full 3 months before improvements were more often seen. However, the significance of these changes and benefits from the tension night splint remain limited.

\section{Possible limitations, and considerations for the future}

Although both groups had improvements seen across several of the outcome measures studied, there were limited statistical differences seen between the groups. A larger study population may be better able to explore this and this could be the focus of further study. In addition, this was a single-blinded study, in which the participants but not the examiner knew which group they had been allocated to. This was chosen for pragmatic purposes, but could have had an influence on the outcome. Further work could be done with sham devices should funding be available to study this as an intervention. Furthermore, this study only used a single commercially available device, and it is not clear if other commercially available devices or custom-made devices have similar outcomes, and if any differences exists between them, and this could be the area of further investigation. Interobserver error is recognised as a limitation for both the goniometer and ultrasound measures, which was avoided with a single investigator taking all of the measurements. Intraobserver error was minimised with familiarity with the techniques used, but could not be eliminated completely and remains a recognised limitation of this study.

Improvements in pain occurred in both groups without necessarily seeing statistically significant differences seen in measures of physical flexibility. This may be that the study was underpowered to detect small changes in flexibility reliably, and either larger groups or more robust methods are needed. Alternatively, it may be that the improvements seen represent changes other than that directly from changes in flexibility, such as improvements in the load tolerability of tissue, or desensitisation of tissue, and exploring these areas could be the focus of further work.

\section{Summary}

Overall this study has shown that even in patients with very chronic symptoms of an average of 2 years, improvements in pain and function can be found using a structured HEP. However, from the data seen here, it appears that the addition of a TNS does not have much, if any, benefit beyond this in the treatment of this population.

Acknowledgements The author would like to offer his thanks to Chloe Tattersall, the nurse practitioner in the Sports Medicine Department at University Hospitals of Leicester for conducting the study blinding. The author would also like to offer his thanks to Dr Jonathan Folland at Loughborough University for his support during the study, and to the Leicester Arthritis Research Fund for the small charitable grant that allowed the purchase of the tension night splint devices.

Contributors This manuscript has a sole author who was responsible for the design, setting up and analysis of this study.

\section{Competing interests None declared.}

Patient consent Not required in this study design fully anonymised data used only no specific individuals can be identified from material used patients consented to enter study, and for anonymised data from this to be used to disseminate in medical journals.

Ethics approval NHS Research Ethics Committee (REC ref 14/WS/1069).

Provenance and peer review Not commissioned; externally peer reviewed.

Open Access This is an Open Access article distributed in accordance with the Creative Commons Attribution Non Commercial (CC BY-NC 4.0) license, which permits others to distribute, remix, adapt, build upon this work noncommercially, and license their derivative works on different terms, provided the original work is properly cited and the use is non-commercial. See: http:// creativecommons.org/licenses/by-nc/4.0/

(C) Article author(s) (or their employer(s) unless otherwise stated in the text of the article) 2017. All rights reserved. No commercial use is permitted unless otherwise expressly granted.

\section{REFERENCES}

1. Buchbinder R. Plantar Fasciitis. New England Journal of Medicine 2004;350:2159-66.

2. Riddle DL, Schappert SM. Volume of ambulatory care visits and patterns of care for patients diagnosed with plantar fasciitis: a national study of medical doctors. Foot Ankle Int 2004;25:303-10. 
3. Orchard J. Plantar fasciitis. BMJ 2012;345:e6603.

4. Lemont $\mathrm{H}$, Ammirati KM, Usen N. Plantar fasciitis: a degenerative process (fasciosis) without inflammation. J Am Podiatr Med Assoc 2003;93:234-7.

5. Puttaswamaiah R, Chandran P. Degenerative plantar fasciitis: A review of current concepts. Foot 2007;17:3-9.

6. Stecco C, Corradin M, Macchi V, et al. Plantar fascia anatomy and its relationship with Achilles tendon and paratenon. J Anat 2013;223:665-76.

7. Snow SW, Bohne WH, DiCarlo E, et al. Anatomy of the Achilles tendon and plantar fascia in relation to the calcaneus in various age groups. Foot Ankle Int 1995;16:418-21.

8. Cheung JT, Zhang M, An KN, . Effect of Achilles tendon loading on plantar fascia tension in the standing foot. Clin Biomech 2006;21:194-203.

9. Wearing SC, Smeathers JE, Urry SR, et al. The pathomechanics of plantar fasciitis. Sports Med 2006;36:585-611.

10. Bolívar YA, Munuera PV, Padillo JP. Relationship between tightness of the posterior muscles of the lower limb and plantar fasciitis. Foot Ankle Int 2013;34:42-8.

11. Irving DB, Cook JL, Menz HB. Factors associated with chronic plantar heel pain: a systematic review. J Sci Med Sport 2006;9:11-22.

12. Riddle DL, Pulisic M, Pidcoe $P$, et al. Risk factors for Plantar fasciitis: a matched case-control study. J Bone Joint Surg Am 2003;85A:872-7.

13. Porter D, Barrill E, Oneacre K, et al. The effects of duration and frequency of Achilles tendon stretching on dorsiflexion and outcome in painful heel syndrome: a randomized, blinded, control study. Foot Ankle Int 2002;23:619-24.

14. Wolgin M, Cook C, Graham C, et al. Conservative treatment of plantar heel pain: long-term follow-up. Foot Ankle Int 1994;15:97-102.

15. DiGiovanni BF, Nawoczenski DA, Lintal ME, et al. Tissue-specific plantar fascia-stretching exercise enhances outcomes in patients with chronic heel pain. A prospective, randomized study. J Bone Joint Surg Am 2003;85-A:1270-7.

16. Hyland MR, Webber-Gaffney A, Cohen L, et al. Randomized controlled trial of calcaneal taping, sham taping, and plantar fascia stretching for the short-term management of plantar heel pain. J Orthop Sports Phys Ther 2006;36:364-71.

17. van de Water AT, Speksnijder CM. Efficacy of taping for the treatment of plantar fasciosis: a systematic review of controlled trials. J Am Podiatr Med Assoc 2010;100:41-51.

18. Landorf KB, Keenan AM, Herbert RD. Effectiveness of foot orthoses to treat plantar fasciitis: a randomized trial. Arch Intern Med 2006;166:1305-10.

19. Wapner KL, Sharkey PF. The use of Night Splints for treatment of Recalcitrant Plantar Fasciitis. Foot Ankle Int 1991;12:135-7.

20. Batt ME, Tanji JL, Skattum N. Plantar fasciitis: a prospective randomized clinical trial of the tension night splint. Clin J Sport Med 1996;6:158-62.

21. Probe RA, Baca M, Adams R, et al. Night splint treatment for plantar fasciitis. A prospective randomized study. Clin Orthop Relat Res 1999;368:190-5.

22. Powell M, Post WR, Keener J, et al. Effective treatment of chronic plantar fasciitis with dorsiflexion night splints: a crossover prospective randomized outcome study. Foot Ankle Int 1998:19:10-18.

23. Martin JE, Hosch JC, Goforth WP, et al. Mechanical treatment of plantar fasciitis. A prospective study. J Am Podiatr Med Assoc 2001;91:55-62.

24. Barry LD, Barry AN, Chen Y. A retrospective study of standing gastrocnemius-soleus stretching versus night splinting in the treatment of plantar fasciitis. J Foot Ankle Surg 2002;41:221-7.

25. Berlet GC, Anderson RB, Davis $\mathrm{H}$, et al. A prospective trial of night splinting in the treatment of recalcitrant plantar fasciitis: the ankle dorsiflexion dynasplint. Orthopedics 2002;25:1273-5.

26. Wheeler PC. The effectiveness and tolerability of tension night splints for the treatment of patients with chronic plantar fasciitis - A case-series study. Int Musculoskelet Med 2014;36:130-6.

27. Acevedo Jl, Beskin JL. Complications of plantar fascia rupture associated with corticosteroid injection. Foot Ankle Int 1998;19:91-7.

28. Sellman JR. Plantar fascia rupture associated with corticosteroid injection. Foot Ankle Int 1994;15:376-81.

29. Crawford F, Atkins D, Young P, et al. Steroid injection for heel pain: evidence of short-term effectiveness. A randomized controlled trial. Rheumatology 1999;38:974-7.

30. Kalaci A, Cakici $\mathrm{H}$, Hapa $\mathrm{O}$, et al. Treatment of plantar fasciitis using four different local injection modalities: a randomized prospective clinical trial. J Am Podiatr Med Assoc 2009;99:108-13.
31. Aqil A, Siddiqui MR, Solan M, et al. Extracorporeal shock wave therapy is effective in treating chronic plantar fasciitis: a metaanalysis of RCTs. Clin Orthop Relat Res 2013;471:3645-52.

32. Speed C. A systematic review of shockwave therapies in soft tissue conditions: focusing on the evidence. Br J Sports Med 2014;48.

33. Lee TG, Ahmad TS. Intralesional autologous blood injection compared to corticosteroid injection for treatment of chronic plantar fasciitis. A prospective, randomized, controlled trial. Foot Ankle Int 2007;28:984-90.

34. Wheeler P. Autologous blood injections for chronic plantar fasciitis a pilot case-series study shows promising results. Int Musculoskelet Med 2013;35:3-7.

35. Wheeler PC. The role of autologous blood injections in the treatment for patients with chronic plantar fasciitis - A case series and longerterm follow-up. Int Musculoskelet Med 2015;37:47-53.

36. Schepsis AA, Leach RE, Gouyca J, et al. Treatment, Surgical results, and review of the Literature. Clinical Orthopaedics and Related Research 1991;266:185-96.

37. Davies MS, Weiss GA, Saxby TS. Plantar fasciitis: how successful is surgical intervention? Foot Ankle Int 1999;20:803-7.

38. Wheeler P, Boyd K, Shipton M. Surgery for patients with Recalcitrant Plantar Fasciitis: good results at Short-, Medium-, and Long-term Follow-up. Orthop J Sports Med 2014;2.

39. Budiman-Mak E, Conrad KJ, Roach KE. The Foot function index: a measure of foot pain and disability. $J$ Clin Epidemiol 1991;44:561-70.

40. SooHoo NF, Samimi DB, Vyas RM, et al. Evaluation of the validity of the Foot function index in measuring outcomes in patients with foot and ankle disorders. Foot Ankle Int 2006;27:38-42.

41. Budiman-Mak E, Conrad K, Stuck R, et al. Theoretical model and Rasch analysis to develop a revised Foot function index. Foot Ankle Int 2006;27:519-27.

42. Dawson J, Doll H, Coffey J, et al. Responsiveness and minimally important change for the Manchester-Oxford foot questionnaire (MOXFQ) compared with AOFAS and SF-36 assessments following surgery for hallux Valgus. Osteoarthritis Cartilage 2007;15:918-31.

43. Dawson J, Boller I, Doll H, et al. Responsiveness of the ManchesterOxford foot questionnaire (MOXFQ) compared with AOFAS, SF-36 and EQ-5D assessments following foot or ankle surgery. Bone Joint J 2012;94-B:215-21.

44. Morley D, Jenkinson C, Doll H, et al. The Manchester-Oxford Foot Questionnaire (MOXFQ): Development and validation of a summary index score. Bone Joint Res 2013;2:66-9.

45. Morley D, Jenkinson C, Doll H, et al. The Manchester-Oxford Foot Questionnaire (MOXFQ). Development and validation of a summary index score 2013;2:66-9.

46. Martin RL, Irrgang JJ, Burdett RG, et al. Evidence of validity for the Foot and Ankle Ability measure (FAAM). Foot Ankle Int 2005;26:968-83.

47. Herdman M, Gudex C, Lloyd A, et al. Development and preliminary testing of the new five-level version of EQ-5D (EQ-5D-5L). Qual Life Res 2011;20:1727-36.

48. Zigmond AS, Snaith RP. The hospital anxiety and depression scale. Acta Psychiatr Scand 1983;67:361-70.

49. Bjelland I, Dahl AA, Haug TT, et al. The validity of the Hospital anxiety and depression Scale. an updated literature review. $J$ Psychosom Res 2002:52:69-77.

50. Sayar K, Arikan M, Yontem T. Sleep quality in chronic pain patients. Can J Psychiatry 2002;47:844-8.

51. Beaudreau SA, Spira AP, Stewart A, et al. Validation of the Pittsburgh Sleep Quality Index and the Epworth Sleepiness Scale in older black and white women. Sleep Med 2012;13:36-42.

52. Freynhagen R, Baron R, Gockel U, et al. painDETECT: a new screening questionnaire to identify neuropathic components in patients with back pain. Curr Med Res Opin 2006;22:1911-20.

53. Prout MK, Thacker MA, Barnard KJ, et al. Screening for neuropathic low back pain using a validated neuropathic pain questionnaire. J Bone Joint Surg Br 2010;92-B.

54. Gauffin J, Hankama T, Kautiainen $\mathrm{H}$, et al. Neuropathic pain and use of PainDETECT in patients with Fibromyalgia: a cohort study. BMC Neurol 2013;13.

55. Wheeler PC. Neuropathic pain may be common in chronic lower limb tendinopathy: a prospective cohort study. Br J Pain 2017;11:16-22.

56. Landorf KB. Plantar heel pain and plantar fasciitis. BMJ Clin Evid 2015;2015.

57. Mohseni-Bandpei MA, Nakhaee M, Mousavi ME, et al. Application of ultrasound in the assessment of plantar fascia in patients with plantar fasciitis: a systematic review. Ultrasound Med Biol 2014:40:1737-54. 
58. Fabrikant JM, Park TS. Plantar fasciitis (fasciosis) treatment outcome study: plantar fascia thickness measured by ultrasound and correlated with patient self-reported improvement. Foot 2011;21:79-83.

59. Lee WCC, Wong WY, Kung E, et al. Effectiveness of adjustable dorsiflexion night splint in combination with accommodative foot orthosis on plantar fasciitis.

The Rehabil of Rehabilitation Research and Development 2012;49:1557-64.

60. Landorf KB, Radford JA. Minimal important difference: Values for the Foot Health Status Questionnaire, Foot Function Index and Visual Analogue Scale. Foot 2008;18:15-19. 